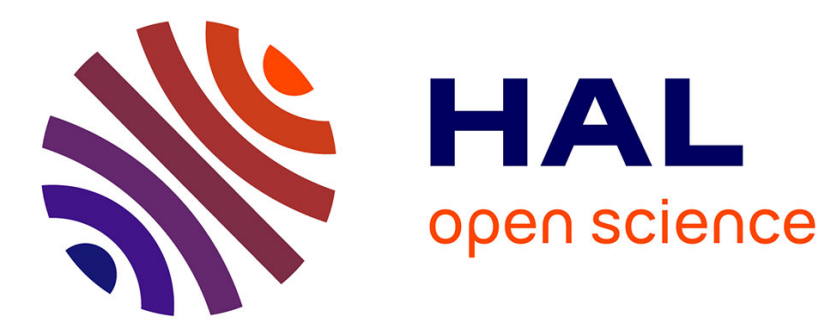

\title{
Dissolution measurement free from mass transport
} Jean Colombani

\section{To cite this version:}

Jean Colombani. Dissolution measurement free from mass transport. Pure and Applied Chemistry, 2013, 85 (1), pp.61-70. 10.1351/PAC-CON-12-03-07 . hal-03229746

\section{HAL Id: hal-03229746 \\ https://hal.science/hal-03229746}

Submitted on 1 Jun 2021

HAL is a multi-disciplinary open access archive for the deposit and dissemination of scientific research documents, whether they are published or not. The documents may come from teaching and research institutions in France or abroad, or from public or private research centers.
L'archive ouverte pluridisciplinaire HAL, est destinée au dépôt et à la diffusion de documents scientifiques de niveau recherche, publiés ou non, émanant des établissements d'enseignement et de recherche français ou étrangers, des laboratoires publics ou privés. 
Pure Appl. Chem., Vol. 85, No. 1, pp. 61-70, 2013.

http://dx.doi.org/10.1351/PAC-CON-12-03-07

(C) 2012 IUPAC, Publication date (Web): 31 August 2012

\title{
Dissolution measurement free from mass transport ${ }^{\star}$
}

\author{
Jean Colombani ${ }^{\ddagger}$ \\ Laboratoire de Physique de la Matière Condensée et Nanostructures, Université \\ de Lyon, Université Claude Bernard Lyon 1, CNRS, UMR 5586, Domaine \\ scientifique de la Doua, F-69622 Villeurbanne cedex, France
}

\begin{abstract}
Standard dissolution experiments are carried out in stirred liquid. Therefore, the measured dissolution rate contains also diffusive and convective contributions, besides the desired surface reaction contribution. We present here a methodology, based on the hydrodynamical analysis of classical dissolution experiments, enabling us to extract the pure dissolution rate constant from the set of the already measured dissolution rates. The application of this analysis to the case of gypsum shows that the removal of the mass transport contribution from the dissolution rates of this mineral found in the literature brings, despite their apparent inconsistency, a coherent description of its dissolution kinetics and a well-defined value of its rate constant.
\end{abstract}

Keywords: aqueous solutions; diffusion; dissolution; experimental methods; geochemistry; interfaces; kinetics; reaction kinetics; surface chemistry.

\section{INTRODUCTION}

The knowledge of the kinetics of mineral-water and organic-water interactions is needed in a large number of processes [1]. Some of these processes, as much natural as industrial, have a strong influence on crucial social issues. For instance, mineral dissolution is involved in at least two major stages of the carbon cycle: the weathering of terrestrial carbonates, and the dissolution of deep-sea sediments, these two processes playing a leading role in controlling the $\mathrm{pH}$ of the ocean, and consequently the atmospheric $\mathrm{pCO}_{2}$ [2,3]. Dissolution and precipitation are also key features of $\mathrm{CO}_{2}$ sequestration in deep aquifers. One can also mention the quality of drinking water, where dissolution drives the transfer of pollutant from soils to water [4]. Besides these geological situations, dissolution determines also the bioavailability of pharmaceutical compounds inside the human body [5].

Reliable dissolution experiments are therefore required to understand and modelize dissolution in situ measurements. Numerous devices are commonly used to access the dissolution kinetics, at the macroscopic (batch dissolution, rotating disk, flowing cell, ...) as much as the nanometric scale (vertical scanning interferometry, atomic force microscopy). But recent reviews have evidenced long-lasting inconsistencies between laboratory dissolution rates, particularly for soft minerals. Arvidson et al. have noticed a 3 orders of magnitude spreading of the dissolution rates of calcite at $\mathrm{pH} \simeq 10$ [6]. Luttge et al. have evidenced a 3 to 4 orders of magnitude discrepancy of the dissolution rates of dolomite at $\mathrm{pH}=3$ [7]. In the case of albite at $\mathrm{pH}=9$, one cannot only find a dispersion of 2 orders of magnitude between dissolution rates in various experiments, but also a disagreement of the evolution of the rate with the

\footnotetext{
*Pure Appl. Chem. 85, 1-305 (2013). A collection of invited papers based on presentations at the $32^{\text {nd }}$ International Conference on Solution Chemistry (ICSC-32), La Grande Motte, France, 28 August-2 September 2011.

‡E-mail: Jean.Colombani@univ-lyon1.fr
} 
distance from equilibrium [8]. For magnesite at $5<\mathrm{pH}<7$, a factor of 10 is observed between macroscopically and nanoscopically measured dissolution rates [9]. These disagreements help to explain why, in the case of calcite for instance, a comprehensive theoretical model of dissolution is still lacking [10].

Much of the origin of these discrepancies lies in the variety of the devices used to access the dissolution rates. In none of the setups, the solvent is at rest. In general, the mineral dissolves in the flowing solvent, and the dissolution rate is deduced from the increase of the concentration in the solvent. So obviously the measured rate derives from a combination of the rate of chemical reaction at the solid-liquid interface (pure dissolution) and of the diffusion velocity across the diffusional boundary layer developing in the vicinity of the interface when placed in a flow (mass transport). The advection of the dissolved species from the upper boundary layer to the concentration measurement device can be neglected because the forced convection (stirring, laminar flow, shaking) is supposed to be intense enough to guarantee a uniform value of the concentration in the bulk liquid. The two events are consecutive so the slowest one leads the kinetics. When mass transport is much slower than the chemical reaction, it drives the whole kinetics, one speaks of a diffusion-controlled situation and no information about the dissolution can be brought from the experiment. When the chemical reaction is much slower than mass transport, it drives the whole kinetics, one speaks of a surface-controlled situation and in this case the measured dissolution rate is directly the chemical reaction rate. But unfortunately, for soft minerals (like sulfates or carbonates) or drugs, both phenomena have a comparable influence and the measured dissolution rate contains intricate information about both kinetics. In the worst case, the rate is given as such and a rigorous use of its value is difficult, because it reflects the specific convective situation of the device (flow field, flow velocity, reactor geometry, ...), not the proper behavior of the mineral. In the best case, an experimental parameter is varied, for instance, the rotation speed in rotating disk experiments, and the pure dissolution value is extrapolated to a zero-convection value.

In this paper, following a methodology introduced in ref. [11], I determine to which extent it is possible to extract the pure dissolution rate constant from a set of literature experiments where the dissolution rate is blurred by diffusion and convection effects. To achieve this goal, a boundary layer analysis is carried out, which enables us to delineate the contribution of the two phenomena to the overall rate, and then isolate the surface reaction rate. Then the rate deduced from the analysis is compared to the rate measured in a non-standard experiment, performed in a non-flowing solvent, where no hydrodynamical assumption is required. The agreement between the two values validates the analysis.

\section{HYDRODYNAMICAL ANALYSIS}

In standard solution chemistry experiments, the observable dissolution flux $J$ is the ultimate consequence of a three-step process:

1. First, ions are hydrated by the solvent and detached from the mineral, due to the difference of the chemical potential of the solid and liquid. I do not attach any importance here to the atomistic mechanism leading to this matter removal (molecular step migration, etch pit deepening, ...) and just consider the phenomenological relation between the thermodynamic force and matter flux [12]. Several chemical models of surface reaction exist and are still under discussion, which provide various rate laws, among which we can cite the transition-state theory (TST) [13] and the step-wave model [14]. The discussion of these models is beyond the scope of this article. Thence, for the demonstration we have made use of a simple linear law between the force (the undersaturation) and the flux $J_{\text {reac }}$, which can be deduced from the TST as much as from the asymptotic behavior of the stepwave model far from equilibrium 


$$
J_{\text {reac }}=k\left(1-\frac{c_{\text {surf }}}{c_{\text {sat }}}\right)
$$

In this equation, $c_{\text {surf }}$ is the concentration of the dissolved species at the solid-liquid interface, $c_{\text {sat }}$ their concentration at chemical equilibrium, and $k$ the surface reaction rate constant.

2. In every kind of experiment, the solution is flowing along a reacting solid, continuous source of matter. Therefore, a diffusional boundary layer develops in the immediate vicinity of the surface, joining the chemical flux of dissolved species at the interface $J_{\text {reac }}$ due to the dissolution, to the convective flux of these species in the bulk liquid, due to the forced convection. This transport boundary layer corresponds to the area where viscous effects are non-negligible, whereas the viscosity is considered as being zero outside this layer [15]. In this area, concentration migrates by diffusion and the matter flux $J_{\text {diff }}$ obeys Fick's law

$$
J_{\text {diff }}=D \frac{c_{\text {surf }}-c_{\text {bulk }}}{\delta}
$$

In this equation, $D$ is the diffusion coefficient of the dissolved species, $c_{\text {bulk }}$ the concentration in the bulk flowing solvent, and $\delta$ the diffusional boundary layer thickness. A linear concentration gradient has been postulated for simplicity. The layer develops from the edge of the solid facing the fluid flow, thickening with the distance $x$ from this edge. An estimate of the thickness $\delta$ of the diffusional boundary layer can be immediately obtained in writing that this thickness is a diffusion length: $\delta \sim \sqrt{D t}$, with $t$ the time. Exiting the layer, the species are carried away by the flow of velocity $v$. At time $t$, the position attained by a fluid particle is $x \sim v t$. Therefore, one gets $\delta \sim \sqrt{D x / v}$ [15]. To use a more quantitative expression subsequently, it has been considered (except for the rotating disk device) that the problem always comes down to a laminar flow along a semi-infinite plate, whatever the geometry of the solid. In this case, the boundary layer thickness writes [16]

$$
\delta=\left(\frac{2 \pi}{0.244}\right)^{1 / 3} v^{1 / 6} v^{-1 / 6} D^{1 / 3} x^{1 / 2}
$$

with $v$ the kinematic velocity of the solution.

3. Finally, the ions reach the diffusional boundary layer surface and are advected by the flow. In the bulk liquid the concentration $c_{\text {bulk }}$ is supposed to be kept uniform by the forced convection, which avoids one to solve Euler's equation to compute the concentration field in the whole cell. Thereby, we consider that the concentration is instantaneously advected from the outside boundary layer to the measurement device.

Therefore, the measured rate $J$ derives from two consecutive timescales. In the case of hard minerals (silicates, aluminates, ...), the reacting step is extremely slow ( $t \sim$ year). Hence, the chemical reaction drives the kinetics and the other stage can be considered as quasi-instantaneous. Therefore, one can merely write $J=J_{\text {reac }}$ and the pure dissolution rate constant $k$ is computed (see, e.g., [17]). In the case of softer minerals, the chemical and transport fluxes are known to be of the same order of magnitude and such an approximation is no more valid (see, e.g., [18]). To access $J_{\text {reac }}$ in this case, a detailed analysis of the interplay between the two fluxes is required. The first step is to take into account mass conservation between the bottom and the top of the diffusional boundary layer. It takes the form of an equality between the mass flow rates at the solid-liquid interface $s_{\mathrm{r}} J_{\text {reac }}\left(s_{\mathrm{r}}\right.$ being the reactive surface area) and at the outside boundary layer $s J_{\text {diff }}(s$ being the surface area of the outside boundary layer, in general identified as the geometrical surface area of the solid). In the expression of these two flow rates, the only unknown parameter is the concentration at the interface $c_{\text {surf }}$, which can therefore be deduced 


$$
c_{\text {surf }}=\frac{s_{\mathrm{r}} k \delta c_{\mathrm{sat}}+s c_{\mathrm{sat}} D c_{\mathrm{bulk}}}{s c_{\mathrm{sat}} D+s_{\mathrm{r}} k \delta}
$$

In all the experiments, the observable dissolution rate $J$ is deduced from the instantaneous increase of $c_{\text {bulk }}$ with time, i.e., of the concentration at the outside boundary layer. So the observable rate corresponds to the rate at this place, and $J=J_{\text {diff }}$. Introducing eq. 4 in this expression, one gets the measured dissolution rate

$$
J=\frac{1}{\frac{s}{s_{\mathrm{r}} k}+\frac{\delta}{D c_{\mathrm{sat}}}}-\frac{c_{\text {bulk }}}{\frac{s c_{\mathrm{sat}}}{s_{\mathrm{r}} k}+\frac{\delta}{D}}
$$

From this equation, we see that the analysis predicts a linear evolution of the overall flux with the bulk concentration. The inverse of the slope of $J\left(c_{\text {bulk }}\right)$ is

$$
\frac{1}{\left(\frac{\partial J}{\partial c_{\text {bulk }}}\right)}=-\frac{c_{\text {sat }}}{\xi_{k}}-\frac{\delta}{D}
$$

where we have introduced the roughness factor $\xi=s_{\mathrm{r}} / s$. From this we see that, provided that we have $1 /\left(\partial J / \partial c_{\text {bulk }}\right)$ data for a range of $\delta$ values, the pure surface reaction rate constant can be obtained from

$$
k=-\frac{c_{\text {sat }}}{\xi \lim _{\delta \rightarrow 0} \frac{1}{\left(\frac{\partial J}{\partial c_{\text {bulk }}}\right)}}
$$

\section{APPLICATION OF THE ANALYSIS TO THE LITERATURE RESULTS OF ONE MINERAL}

To validate this analysis, I had to apply it to the wealth of data accumulated along the decades about one mineral, and find if it provides a unified view of its dissolution kinetics. The choice of the mineral for this demonstration was a crucial point. The solid must exhibit a dissolution rate high enough to be comparable to the diffusion rate (mixed kinetics) and to be measurable in reasonable times, and the chemistry of the reaction should be simple enough to permit one to focus on the hydrodynamics of the experiments. Gypsum $\left(\mathrm{CaSO}_{4}, 2 \mathrm{H}_{2} \mathrm{O}\right)$ has been found to meet the three criteria. Furthermore, experiments have been performed on gypsum in various communities (geochemistry, crystal growth, soil science, oceanography, ...), guaranteeing a broad spectrum of device geometries. Finally, the order of its dissolution rate equation has been found as being one, as in eq. 1 [19]. Figure 1 shows an as exhaustive view as possible of the dissolution rate measurements of gypsum in water found in the literature. One faces a wide dispersion of the rate values, and no coherent vision of the dissolution kinetics emerges from this figure. My aim now is to apply the analysis described in the preceding section to prove quantitatively that this dispersion of the results stems from the variety of the hydrodynamical configurations of the employed devices, which hides the real dissolution kinetics. So every experimental setup has to be analyzed in detail to check the applicability of the method, i.e., mainly the possibility to evaluate the flow velocity and the thickness of the transport boundary layer:

1. The rotating disk is the only technique the hydrodynamics of which has been studied, and used, rigorously. Indeed, it is the only case where the geometry is well defined enough to permit to solve the fluid equations adequately. This computation has been done for long and the diffusional boundary layer thickness is: $\delta=1.61 D^{1 / 3} v^{1 / 6} \omega^{-1 / 2}$ with $v$ the kinematic viscosity of the solution and $\omega$ the angular velocity of the disk [20]. This equation has been used for all rotating disk experiments. One can notice that $\delta$ is independent of the disk dimension. 
2. The most common device of dissolution experiments is merely a reactor containing the stirred solvent in which the mineral in powder dissolves. In this case, the exact shape of the solid is variable among the powder grains. Sometimes, some sample grains are observed with a microscope to estimate the mean shape of the grains [21,22]. Here, to make an at least crude estimate of $\delta$, a strong assumption has to be made. The model of boundary layer along a semi-infinite plate described above is considered to be applicable to all the solid particles of the powder. In other words, the particular shape of the grains is not considered to differ enough from a plate geometry to make eq. 3 nonapplicable. This assumption will be verified a posteriori. One must now identify the quantities in eq. 3 . The flow velocity field in the vessel can be extremely complex and is, at least, not uniform at all (see, e.g., [23]). Therefore, $v$ was chosen as one-third of the speed of the extremity of the stirrer, a value representative of the situation of the flow between the stirrer and the walls [15]. This value is not supposed to be, for instance, the mean value of the flow in the batch, but a value representative of the flow velocity whatever the vessel geometry. The stirrer speed writes $v_{\text {stirrer }}=R \omega$ with $R$ the stirrer radius and $\omega$ the rotation speed. Whereas $\omega$ is systematically stipulated, I had to estimate $R$ sometimes from sketches or descriptions of the setup. The length $x$ over which the boundary layer develops is taken as the average size of the powder grains. This information is always given. In the proposed framework, the radius of a spherical grain is considered as comparable to the length of a grain with a semi-infinite plate shape. Despite the crudeness of the approximation, it captures the main feature of the boundary layer.

3. A combination of the two preceding devices is also encountered, called "mixed reactor" [24], in which a disk of the mineral is rotated at constant speed and the solvent is continuously fed into the reactor. In this case, the analysis is strictly the same as in the simple rotating disk device. The only difference is that the concentration in the reactor depends both on the input concentration and on the dissolution velocity, which changes nothing, provided that this bulk concentration is known.

4. Another device of the same family is the shaken tube setup. Here, the powder and the solvent are sealed in tubes, which are stirred in a centrifuge. I have chosen the flow velocity as the speed of the tube. This one is derived again from $v_{\text {tube }}=R \omega$, with $R$ the radius of the centrifuge plate and $\omega$ the rotation speed. If $\omega$ is always given, $R$ has sometimes been deduced from the centrifuge manufacturer data. Again, this velocity gives only an order of magnitude of the flow speed in the tube, sufficient to capture the main feature of the boundary layer. For $x$, the average size of the grains has again been chosen.

5. The flow cell device is another popular setup of dissolution measurements. In this experiment, the pure solvent is flowing past a bed of mineral samples. The concentration at the end of the cell is measured as a function of time. Unfortunately, in the course of an experiment, the concentration in the cell is neither stationary, nor uniform. It increases both in time (due to the dissolution along the flow) and space (from the solid to the top of the liquid, and from the beginning to the end of the cell). And the resulting non-steady concentration profile is not known. Due to this feature, the dissolution flow rate cannot be deduced from the evolution of the concentration at the exit of the cell. For these reasons, a $J\left(c_{\text {bulk }}\right)$ curve cannot be drawn and a surface reaction rate constant cannot be deduced from flowing cell devices. To circumvent this limitation, Mbogoro et al. have proposed recently a new microdevice of this type, where the whole concentration field in the cell is computed with a finite elements fluid mechanics software $[25,26]$. Whereas this computation lies on hydrodynamical assumptions, it permits a more rigorous analysis of the results, and the pure dissolution rate constant of gypsum they deduce agrees well with the value obtained in next section.

6. Finally, in flume experiments, a solid gypsum sample is placed at the entrance of a broad channel, and the composition in dissolved gypsum of the flowing liquid is measured downstream in the channel. The variation of the dissolution rate is studied as a function of the liquid flow rate. 


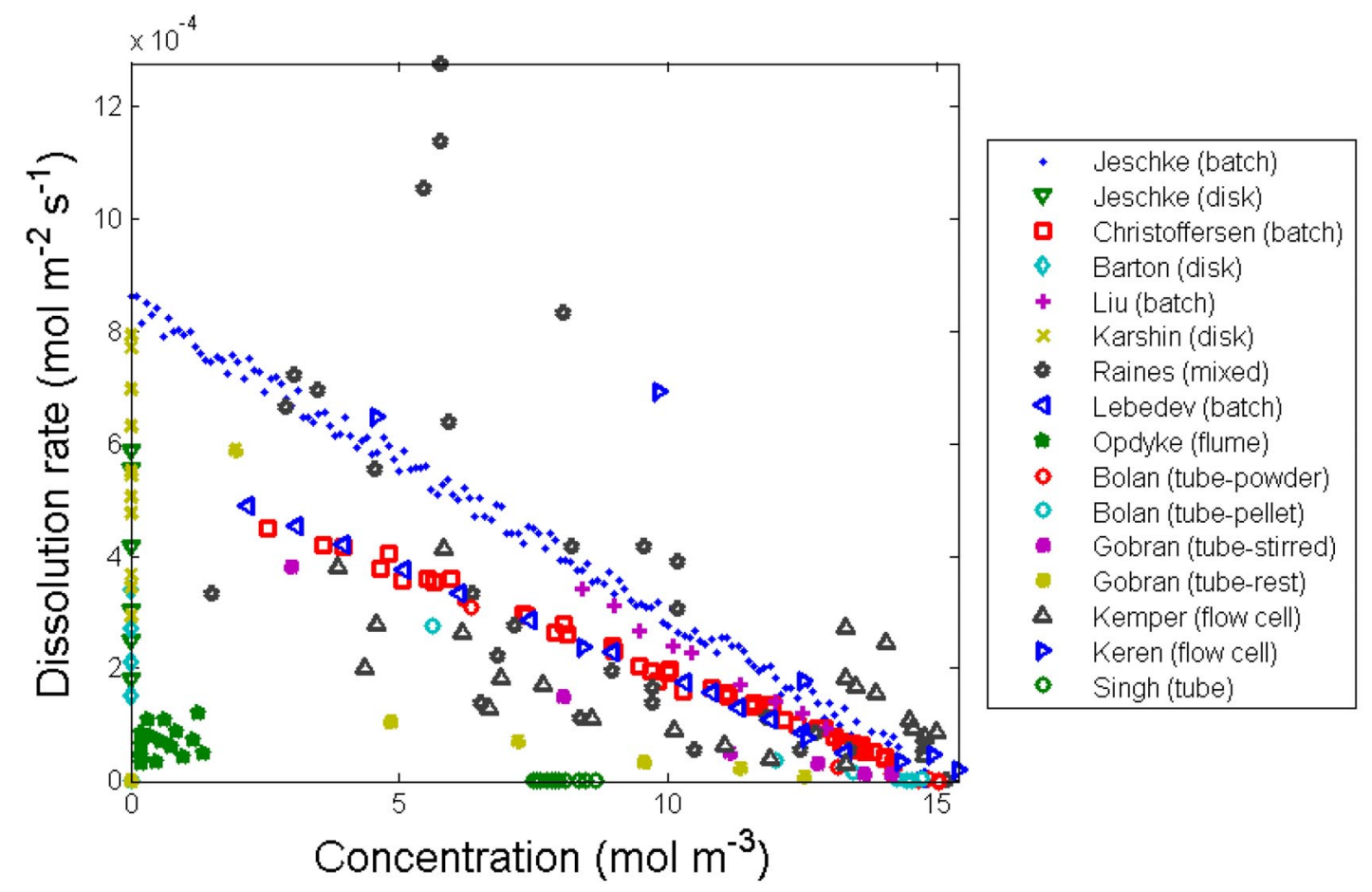

Fig. 1 Dissolution rate $J$ of gypsum in water as a function of calcium bulk concentration $c_{\text {bulk }}$ for the stirred batch experiments by Jeschke et al. [21], Christoffersen and Christoffersen [22], Liu and Nancollas [35], and Lebedev and Lekov [36], from rotating disk experiments by Jeschke et al. [21], Barton and Wilde [20], and Karshin and Grigoryan [37], from mixed flow/rotating disk reactor experiments by Raines and Dewers [24], from shaken tube experiments by Bolan et al. [38] (with powder or compressed powder pellets), Gobran and Miyamoto [39], and Singh and Bajwa [31], from flow cell experiments by Kemper et al. [29] and Keren and O'Connor [30] and flume experiments by Opdyke et al. [40].

The liquid velocity is directly given in the article, and the boundary layer thickness was computed with eq. 3, with $x$ as the length of the solid along the flow.

We dispose now of an estimate of the diffusional boundary layer thickness for every setup (except the unusable flow cell experiments). We need also ( $\partial J / \partial c_{\text {bulk }}$ ) for each device. To get it, two possibilities exist. Either a $J\left(c_{\text {bulk }}\right)$ experimental curve exists for a given $\delta$ value (batch, shaken tube). In this case, the curve is considered to be linear, which is in general not far from the reality, and the slope of the straight line is merely computed. Or $J\left(c_{\text {bulk }}\right)$ is known for a given $c_{\text {bulk }}$ (often $\left.c_{\text {bulk }}=0\right)$ and various $\delta$ (rotating disk, mixed reactor, flume). In this case, a linear law is postulated and $\left(\partial J / \partial c_{\text {bulk }}\right)=$ $\left[0-J\left(c_{\text {bulk }}\right)\right] /\left(c_{\text {sat }}-c_{\text {bulk }}\right)$.

Besides the diffusion coefficient of the dissolved species of gypsum in water has been measured by holographic interferometry: $D=1.0 \times 10^{-9} \mathrm{~m}^{2} \mathrm{~s}^{-1}$ [27]. The gypsum solubility in water is known for long: $c_{\text {sat }}=15 \mathrm{mmol} / \mathrm{kg}=2 \mathrm{~g} / \mathrm{L}$ [28]. The last problem is the access to the roughness factor $\xi$. Unfortunately, the reactive surface area $s_{\mathrm{r}}$ and the geometric surface area $s$ are almost never indicated in the articles. Only one paper provides these two quantities, from which a roughness factor of $\xi=$ $1100 / 60=18.3$ can be computed [21]. In the absence of other data, this value has been used for all devices, hoping that the grinding of gypsum into powder always leads to a similar $\xi$ value. 


\section{RESULTS}

Before using this analysis to access to the pure dissolution rate constant, I have made use of it to get information on the hydrodynamical state of the various devices. Figure 2 shows the boundary layer thickness as a function of the length of the investigated samples for the batch and shaken tube experiments. We observe that the thicknesses probed by the various setups span from 2 to $20 \mu \mathrm{m}$, varying mostly with the flow rate resulting from the stirring.

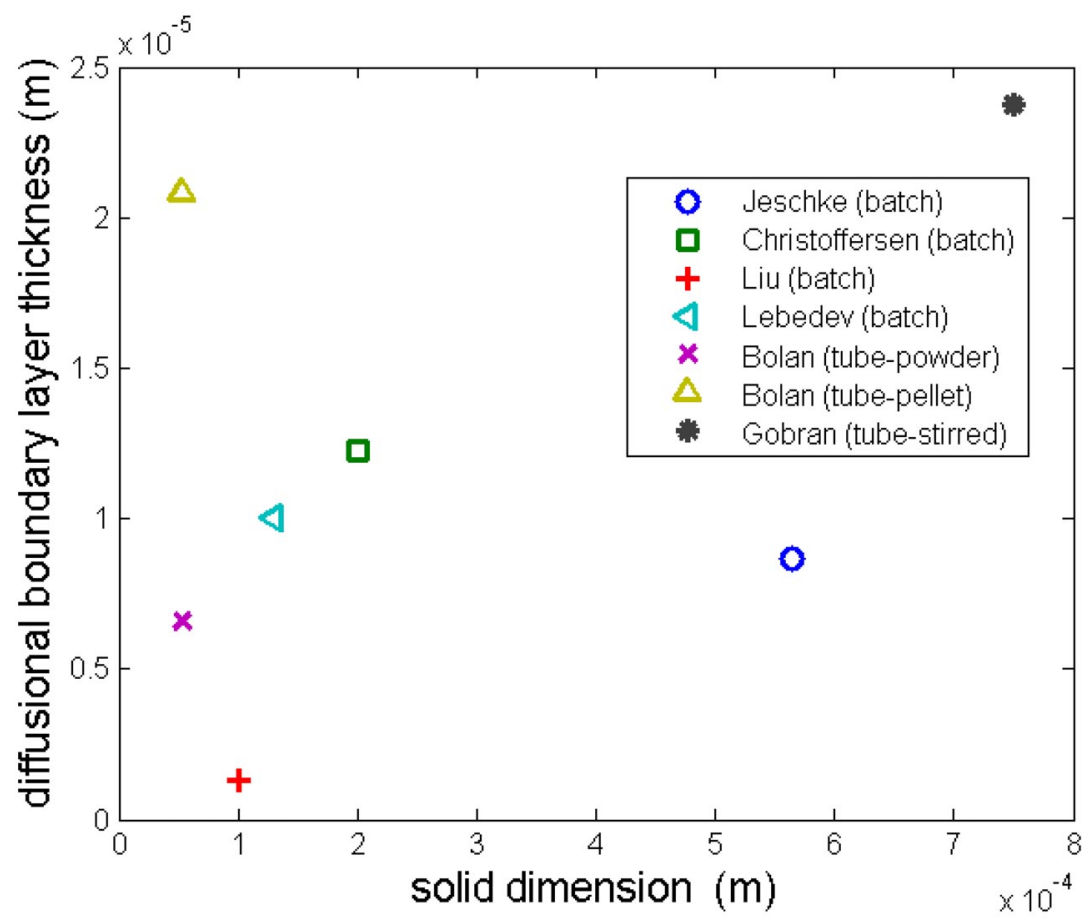

Fig. 2 Mass transport boundary layer thickness $\delta$ as a function of the dimension of the dissolving solid $x$ (powder grain, pellet) for the stirred batch experiments [21,22,35,36] and shaken tube experiments [38,39].

We have now all the elements in hand to compute the pure dissolution rate constant from the results of all experiments via eq. 7 . Figure 3 collects the values of $1 /\left(\partial J / \partial c_{\text {bulk }}\right)$ for each experiment as a function of the boundary layer thickness. As predicted by eq. 6 , all these data build a straight line, the extrapolation of which to $\delta=0$ enables to compute $k$ via eq. $7: k=7 \times 10^{-5} \mathrm{~mol} \mathrm{~m}^{-2} \mathrm{~s}^{-1}$. As explained in the preceding section, all experiments have been included except the flow cells $[29,30]$, where the $J\left(c_{\text {bulk }}\right)$ curves are not accessible, and the shaken tube experiments of Singh and Bajwa, giving inexplicably rates one order of magnitude lower than all other rates [31]. We see that once the variable mass transport contribution is removed from the observed flux, the set of all setups gives coherent information on the surface reaction kinetics. This agreement between different experimental geometries giving apparently different results validates the hydrodynamical assumptions used for the analysis. Unfortunately, the lack of knowledge of $\xi$ for each experiment prevents the obtainment of an even better convergence of the values of $1 /\left(\partial J / \partial c_{\text {bulk }}\right)$.

To further ascertain this analysis, we have compared this value of $k$ to the one obtained in a nonstandard dissolution experiment: $k=(5 \pm 2) \times 10^{-5} \mathrm{~mol} \mathrm{~m}^{-2} \mathrm{~s}^{-1}$ [27,32]. For this experiment, we had modified a holographic interferometry device initially devoted to fluid mechanics [33], to perform dissolution measurements. The specificity of this method is to be carried out in still water, the sample being 


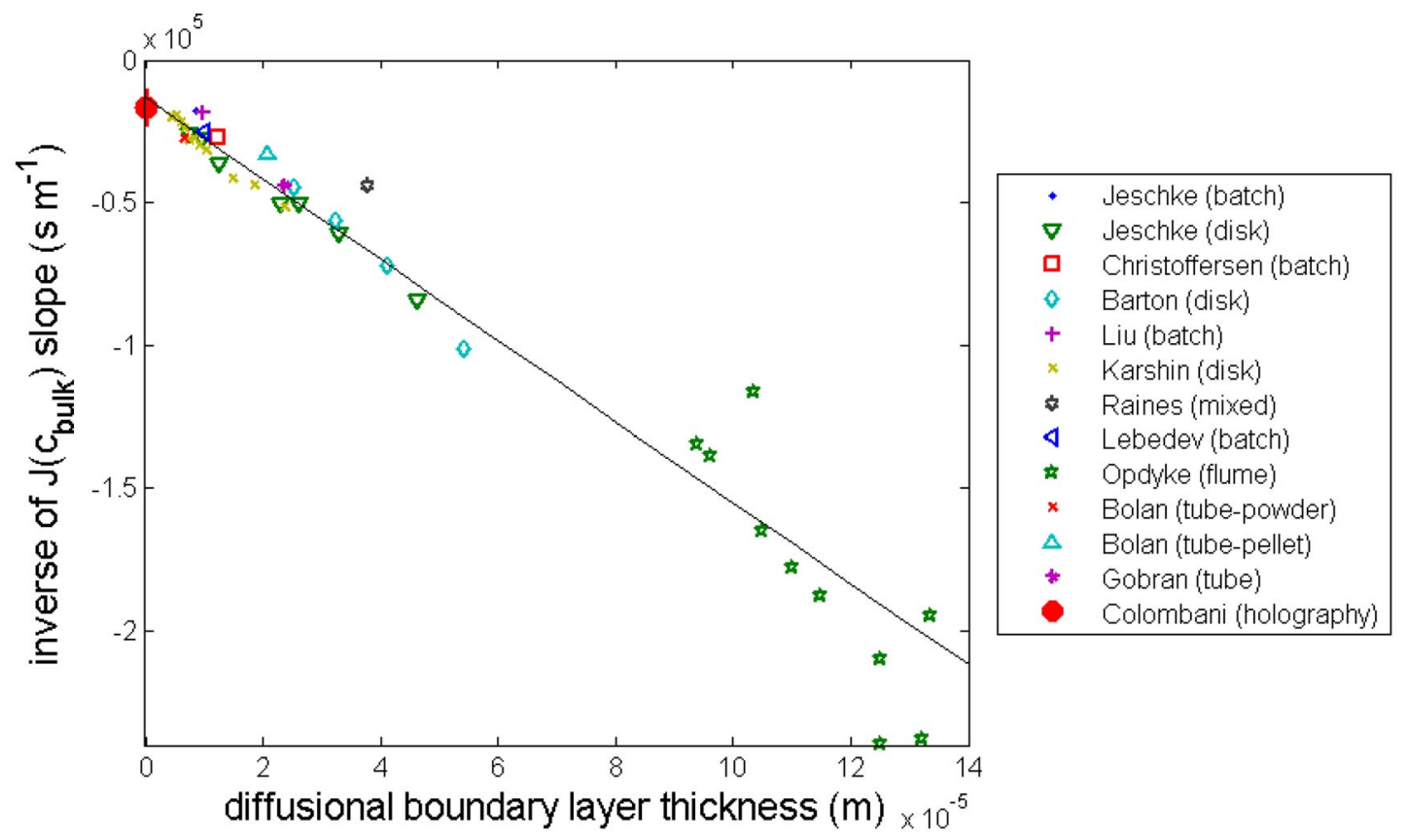

Fig. $31 /\left(\partial J / \partial c_{\text {bulk }}\right)$ for various devices as a function of the boundary layer thickness $\delta$ in each device. The symbols are the same as in Fig. 1. The value at $\delta=0$ corresponds to the value measured by holographic interferometry [27].

simply deposited at the bottom of a cell full of quiescent water. The dissolution of the mineral induces transfer of ions from the solid to the liquid, followed by the migration of the ions upward by simple Fick diffusion. The concentration field in the cell is monitored by holographic interferometry. This field is fitted with its theoretical expression obtained by solving Fick's equation, with the relevant boundary conditions. From the fit, the diffusion coefficient $D$ of dissolved gypsum and the surface reaction rate constant $k$ of gypsum are deduced. The reliability of this measurement comes from the facts that (i) the absence of convective motion avoids the need of a hydrodynamical analysis, like for the other devices, and that (ii) the surface, where the dissolution flux occurs, is directly observed, which avoids the need to compute this local quantity from measurement made far away from the surface, like in standard studies.

The holographic value of $-\xi k / c_{\text {sat }}$ is added in Fig. 3 at $\delta=0$ (because no convection is present). We see that it takes place exactly where the extrapolation of the literature results expected it. This observation constitutes a second validation of the methodology. We can note that the experiments of Raines and Dewers [24] are discrepant from the other ones, which gives credence to the critics made in their comment by Dreybrodt and Gabrovsek [34].

This result is promising in the sense that despite the apparent inconsistency between the data in Fig. 1, a coherent description of the dissolution kinetics of the mineral has been recovered. Furthermore, it appears that the set of all the devices enables one to access to pure dissolution-under the condition that the convective-diffusive contribution is taken into account-because their various geometries probe a broad range of hydrodynamical conditions.

If the correct dissolution behavior can be recovered from the set of all experiments by extrapolation, the question arises to know whether every individual experiment may bring alone a valid information. To check this proposition, the pure dissolution rate constant has been computed via eq. 6 for each device. As can be seen in Fig. 4, the results span over more than one order of magnitude. This dispersion is a proof that the hydrodynamics of all the solution chemistry-type devices is not well defined enough to permit an unquestionable measurement of $k$. To measure a reliable surface reaction rate con- 


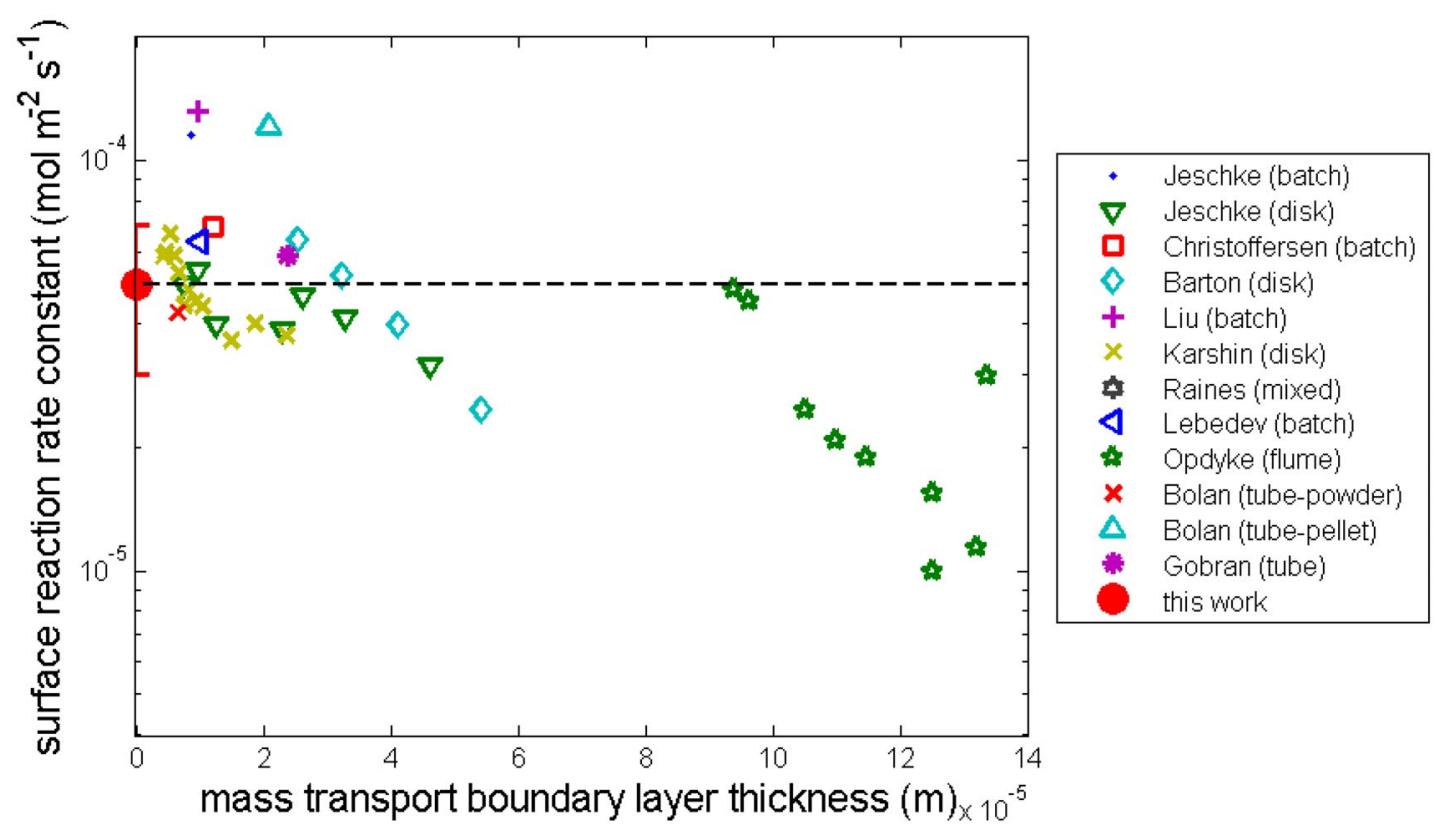

Fig. 4 Logarithm of the pure dissolution rate constant $k$ of gypsum measured with various devices as a function of the diffusional boundary layer thickness $\delta$ for each device. The symbols are the same as in Fig. 1 . The value at $\delta=0$ corresponds to the value measured by holographic interferometry [27].

stant, an averaging over numerous hydrodynamical configurations is needed (like in Fig. 3) or the use of a device using a nonflowing liquid (like in ref. [27]).

\section{CONCLUSION}

I have proposed here a methodology to extract the pure dissolution behavior from dissolution experiments of solution chemistry type, where a powder (or a pellet or a disk) is dissolving in a stirred solvent. To do so, the hydrodynamics of these devices was analyzed, in order to estimate the influence on the dissolution rate of the diffusive and convective transport of the dissolved species. Then these mass transport contributions were removed and the pure surface reaction rate constant computed. This analysis was applied to the case of gypsum, and all the dissolution rates of this mineral available in the literature were collected and analyzed accordingly. Starting from apparently extremely dispersed rates, this analysis permits one to compute a pure dissolution rate constant consistent between all the experiments, which compares perfectly with its value measured with a nonstandard technique, performed in still water. This application to gypsum validates the methodology and opens the way to its application to other soft minerals, where the inconsistency between the literature results has not found explanation yet.

\section{ACKNOWLEDGMENTS}

I thank Luc Petit for fruitful discussions. This work is supported by the Lafarge Centre de Recherche and CNES (French space agency). 


\section{REFERENCES}

1. Jacques Schott, Oleg S. Pokrovsky, Eric H. Oelkers. "The link between mineral dissolution/precipitation kinetics and solution chemistry", in Thermodynamics and Kinetics of Water-Rock Interaction, Vol. 70 of Reviews in Mineralogy \& Geochemistry, E. H. Oelkers, J. Schott, (Eds.), p. 207, Mineralogical Society of America (2009).

2. D. Anderson, D. Archer. Nature 416, 70 (2002).

3. B. Hales, S. Emerson. Earth Planet. Sci. Lett. 148, 317 (1997).

4. R. Kuechler, K. Noack, T. Zorn. Ecol. Model. 176, 1 (2004).

5. D. Mangin, E. Garcia, S. Gerard, C. Hoff, J. P. Klein, S. Veesler. J. Crystal Growth 286, 121 (2006).

6. R. S. Arvidson, I. E. Ertan, J. E. Amonette, A. Luttge. Geochim. Cosmochim. Acta 67, 1623 (2003).

7. A. Luttge, U. Winkler, A. C. Lasaga. Geochim. Cosmochim. Acta 67, 1099 (2003).

8. T. E. Burch, K. L. Nagy, A. C. Lasaga. Chem. Geol. 105, 137 (1993).

9. G. Jordan, O. S. Pokrovsky, X. Guichet, W. W. Schmahl. Chem. Geol. 242, 484 (2007).

10. J. W. Morse, R. S. Arvidson, A. Luttge. Chem. Rev. 107, 342 (2007).

11. J. Colombani. Geochim. Cosmochim. Acta 72, 5634 (2008).

12. E. A. Pachon-Rodriguez, A. Piednoir, J. Colombani. Phys. Rev. Lett. 107, 146102 (2011).

13. H. C. Hegelson, W. M. Murphy, P. Aagaard. Geochim. Cosmochim. Acta 48, 2405 (1984).

14. A. C. Lasaga, A. Luttge. Science 291, 2400 (2001).

15. E. Guyon, J. P. Hulin, L. Petit, C. D. Mitescu. Physical Hydrodynamics, Oxford University Press (2001).

16. F. Jousse, T. Jongen, W. Agterof. Int. J. Heat Mass Transfer 48, 1563 (2005).

17. J. M. Gautier, E. H. Oelkers, J. Schott. Geochim. Cosmochim. Acta 65, 1059 (2001).

18. A. A. Jeschke, W. Dreybrodt. Chem. Geol. 192, 183 (2002).

19. C. F. Fan, H. H. Teng. Chem. Geol. 245, 242 (2007).

20. A. F. M. Barton, N. M. Wilde. Trans. Faraday Soc. 67, 3590 (1971).

21. A. A. Jeschke, K. Vosbeck, W. Dreybrodt. Geochim. Cosmochim. Acta 65, 27 (2001).

22. J. Christoffersen, M. R. Christoffersen. J. Crystal Growth 35, 79 (1976).

23. G. Bai, P. M. Armenante. J. Pharm. Sci. 98, 1511 (2009).

24. M. A. Raines, T. A. Dewers. Chem. Geol. 140, 29 (1997).

25. M. M. Mbogoro, M. E. Snowden, M. A. Edwards, M. Peruffo, P. R. Unwin. J. Phys. Chem. C 115, 10147 (2011).

26. R. D. Fisher, M. M. Mbogoro, M. E. Snowden, M. B. Joseph, J. A. Covington, P. R. Unwin, R. I. Walton. ACS Appl. Mater. Interfaces 3, 3528 (2011).

27. J. Colombani, J. Bert. Geochim. Cosmochim. Acta 71, 1913 (2007).

28. K. U. G. Raju, G. Atkinson. J. Chem. Eng. Data 35, 361 (1990).

29. W. D. Kemper, J. Olsen, C. J. DeMooy. Soil Sci. Soc. Am. Proc. 39, 458 (1975).

30. R. Keren, G. A. O'Connor. Soil Sci. Soc. Am. J. 46, 26 (1982).

31. H. Singh, M. S. Bajwa. Aust. J. Soil Res. 28, 947 (1990).

32. E. A. Pachon-Rodriguez, J. Colombani. AIChE, in press.

33. J. Colombani, J. Bert. Meas. Sci. Technol. 10, 886 (1999).

34. W. Dreybrodt, F. Gabrovsek. Chem. Geol. 168, 169 (2000).

35. S. T. Liu, G. H. Nancollas. J. Inorg. Nucl. Chem. 33, 2311 (1971).

36. A. L. Lebedev, A. V. Lekov. Geochem. Int. 27, 85 (1990).

37. V. P. Karshin, V. A. Grigoryan. Russ. J. Phys. Chem. 44, 762 (1970).

38. N. S. Bolan, J. K. Syers, M. E. Sumner. J. Sci. Food Agric. 57, 527 (1991).

39. G. R. Gobran, S. Miyamoto. Soil Sci. 140, 89 (1985).

40. B. N. Opdyke, G. Gust, J. R. Ledwell. Geophys. Res. Lett. 14, 1131 (1987). 